\title{
Performance Analysis of Multiple Antenna Systems with VQ-Based Feedback
}

\author{
June Chul Roh and Bhaskar D. Rao \\ Department of Electrical and Computer Engineering \\ University of California, San Diego \\ La Jolla, CA 92093-0407, USA \\ Email: jroh@ece.ucsd.edu, brao@ece.ucsd.edu
}

\begin{abstract}
We investigate vector quantization (VQ) methods for feeding back the channel information through a low-rate feedback channel in the context of multiple-antenna MISO systems. The performance of systems with VQ-based quantized beamforming is analyzed for the independent Rayleigh fading case. This requires finding the density of the squared innerproduct between the optimum and the quantized beamforming vector, which is obtained by considering a simple approximation of the quantization cell. The approximate density function is used to lower bound the capacity loss due to quantization, the outage probability and the bit error probability.
\end{abstract}

\section{INTRODUCTION}

The performance achievable using multiple antennas depends on the nature of channel state information (CSI) available at the transmitter and at the receiver. We assume perfect CSI at the receiver and focus on the CSI at the transmitter (CSIT). A higher capacity link can be achieved when the transmitter has perfect CSIT. Other benefits with perfect CSIT include lower complexity receivers and better system throughput in a multiuser environment. However, the assumption that the transmitter has perfect knowledge of multi-dimensional channel is unrealistic, as in many practical systems, CSIT is provided through a finite-rate feedback channel. In this paper, we focus our attention on multiple-input single-output (MISO) systems and on the problem of feeding back the beamforming vector through a finite-rate feedback channel. Recently, systematic methods to analytically quantify the performance of finite-rate feedback systems have begun to appear [1], [2]. Our work is along these lines of inquiry and attempts to further the design methodology and analytical understanding of quantized beamforming systems.

We use the following notations. $A^{\dagger}$ indicates the conjugate transpose of matrix $A$. The inner product between two vectors is defined as $\langle u, v\rangle=u^{\dagger} v$ and the 2-norm of vector $v$ is denoted by $\|v\|=\langle v, v\rangle^{1 / 2}$. $E[\cdot]$ represents the expectation operator, and $\mathcal{N}(\mu, \Sigma)$ and $\tilde{\mathcal{N}}(\mu, \Sigma)$ are respectively the real and the proper complex Gaussian random vector with mean $\mu$ and covariance $\Sigma$. Uniform distribution over a set $S$ is denoted by $\mathcal{U}(S)$. The function $\log (\cdot)$ is the natural logarithm.

This research was supported in part by UC Discovery Grant No. core0210109 sponsored by Ericsson, and in part by the U.S. Army Research Office under MURI Grant No. W911NF-04-1-0224.

\section{SYSTEM MODEL}

We consider a MISO system with $t$ antennas at the transmitter. Assuming flat-fading, the multiple-antenna channel is modeled by the channel vector $h \in \mathbb{C}^{t}$. That is, the channel input $x \in \mathbb{C}^{t}$ and the channel output $y \in \mathbb{C}$ have the following relationship:

$$
y=h^{\dagger} x+\eta
$$

where $\eta$ is the additive white Gaussian noise distributed by $\tilde{\mathcal{N}}(0,1)$. The average transmit power is denoted by $P_{T}$, i.e., $E\left[x^{\dagger} x\right]=P_{T}$. The channel vector will be also written in term of its magnitude and direction as $h=\alpha v$, where $\alpha=\|h\|$ and $v=h /\|h\|{ }^{1}$

It is well-known that, with perfect CSIT, transmit beamforming along $v$ is the optimum choice for maximizing the received SNR and also the mutual information. In this paper, we consider quantization of the unit-norm vector for feedback to the transmitter through a feedback channel. The capacity of the feedback channel is assumed to be finite and limited to $B$ bits per channel update. A quantization codebook $\mathcal{C}=$ $\left\{\hat{v}_{1}, \ldots, \hat{v}_{N}\right\}$, where $\left\|\hat{v}_{i}\right\|=1$ and $N=2^{B}$, is known to both the receiver and the transmitter. Based on the channel information, the receiver selects the best beamforming vector, say $\hat{v}$, from the codebook and sends the corresponding index for the selected beamforming vector to the transmitter. We assume feedback with no error and no delay, and focus on the effect of quantizing the channel information.

At the transmitter, $\hat{v}$ is employed as transmit beamforming vector $(\|\hat{v}\|=1)$. That is, an information-bearing symbol $s \in$ $\mathbb{C}$ is transmitted as $x=\hat{v} s$, resulting in

$$
y=\alpha\langle v, \hat{v}\rangle s+\eta
$$

where $E\left[|s|^{2}\right]=P_{T}$. In this setting, the received SNR is simply given by $\mathrm{SNR}=\alpha^{2}|\langle v, \hat{v}\rangle|^{2} P_{T}$. And the mutual information between $s$ and $y$ for given $h$ and $\hat{v}$ is given by

$$
I(h, \hat{v})=\log \left(1+\alpha^{2}|\langle v, \hat{v}\rangle|^{2} P_{T}\right)
$$

in nats per channel use, where it is assumed that $s \sim$ $\tilde{\mathcal{N}}\left(0, P_{T}\right)$. It is obvious that $I(h, \hat{v})$ with finite $B$ is less than the mutual information with perfect feedback (with $B=\infty$ ),

\footnotetext{
${ }^{1}$ Though not explicitly indicated for notational simplicity, note that throughout the paper $\alpha$ and $v$ (also $\hat{v}$ later) are functions of $h$.
} 
$I(h, v)=\log \left(1+\alpha^{2} P_{T}\right)$, since $|\langle v, \hat{v}\rangle|^{2}<1$. The capacity loss due to quantization of beamforming vector is defined as

$$
C_{L}=E[I(h, v)]-E[I(h, \hat{v})]=E\left[I_{L}(h, \hat{v})\right]
$$

where $E[I(h, v)]$ and $E[I(h, \hat{v})]$ are the ergodic capacities when the transmitter uses the optimum and the quantized beamforming vector, respectively; and $I_{L}(h, \hat{v})=I(h, v)-$ $I(h, \hat{v})$. With a little manipulation, it can be rewritten as

$$
I_{L}(h, \hat{v})=-\log \left(1-\frac{\alpha^{2} P_{T}}{1+\alpha^{2} P_{T}}\left(1-|\langle v, \hat{v}\rangle|^{2}\right)\right) .
$$

\section{QuANTIZATION OF RANDOM BEAMFORMing Vector}

For designing the beamforming codebook, a good design criterion could be to maximize the expected mutual information $E[I(h, \hat{v})]$, or equivalently, to minimize the capacity loss defined in (4). However, unfortunately using it directly leads to difficulty with the centroid computation, an essential step in VQ design (see e.g., [1]). Instead of using the direct form of the capacity loss, an alternative simpler criterion can be derived using an approximation to the capacity loss [3], [4]. We briefly review this approach before proceeding with the analysis.

When $P_{T} \ll 1$, or when $|\langle v, \hat{v}\rangle|$ is close to one (which is valid when $N$ is reasonably large), (5) can be approximated using $-\log (1-x) \simeq x$ for small $x$ as $I_{L}(h, \hat{v}) \simeq \tilde{\alpha}^{2}(1-$ $\left.|\langle v, \hat{v}\rangle|^{2}\right)$ where $\tilde{\alpha}=\sqrt{\frac{\alpha^{2} P_{T}}{1+\alpha^{2} P_{T}}}$. This results in

$$
C_{L} \simeq E\left[\tilde{\alpha}^{2}\left(1-|\langle v, \hat{v}\rangle|^{2}\right)\right]
$$

which is the basis of the quantizer design.

New Design Criterion: Design a quantizer $\mathcal{Q}$ (mathematically, $\mathcal{Q}: \mathbb{C}^{t} \rightarrow \mathcal{C}$ ) to maximize the mean squared weighted inner-product (MSwIP),

$$
\max _{\mathcal{Q}(\cdot)} E|\langle\tilde{\alpha} v, \mathcal{Q}(h)\rangle|^{2}
$$

where $\hat{v}=\mathcal{Q}(h)$ is the quantized beamforming vector $(\|\hat{v}\|=$ 1 ) from codebook $\mathcal{C}$. This VQ design criterion will be called the MSwIP criterion [4].

One of virtues with the MSwIP criterion is that it does lead to a closed-form VQ design algorithm. The design algorithm is similar in nature to the Lloyd algorithm in VQ study which is based on two conditions: the nearest neighborhood condition (NNC) and the centroid condition (CC) [5]. The same approach is used here for designing the quantizer.

New Design Algorithm:

1. NNC: For given code vectors $\left\{\hat{v}_{i} ; i=1, \ldots, N\right\}$, the optimum partition cells satisfy that for $i=1, \ldots, N$,

$$
\mathcal{R}_{i}=\left\{h \in \mathbb{C}^{t}:\left|\left\langle\tilde{\alpha} v, \hat{v}_{i}\right\rangle\right| \geq\left|\left\langle\tilde{\alpha} v, \hat{v}_{j}\right\rangle\right|, \forall j \neq i\right\} .
$$

where $\mathcal{R}_{i}$ is the partition cell (Voronoi region) for the $i$ th code vector $\hat{v}_{i}$. $\tilde{\alpha}$ does not impact the partitioning.

2. $C C$ : For a given partition $\left\{\mathcal{R}_{i} ; i=1, \ldots, N\right\}$, the optimum code vectors satisfy that for $i=1, \ldots, N$,

$$
\begin{aligned}
\hat{v}_{i} & =\arg \max _{\hat{v} \in \mathcal{R}_{i},\|\hat{v}\|=1} E\left[|\langle\tilde{\alpha} v, \hat{v}\rangle|^{2} \mid h \in \mathcal{R}_{i}\right] \\
& =\text { (principal eigenvector) of } E\left[\tilde{\alpha}^{2} v v^{\dagger} \mid h \in \mathcal{R}_{i}\right] .
\end{aligned}
$$

The above two conditions are iterated until $E|\langle\tilde{\alpha} v, \mathcal{Q}(h)\rangle|^{2}$ converges. In practice, a quantizer is designed using a sufficiently large number of training samples (channel realizations). In that case, the statistical correlation matrix in (10) is replaced with a sample average.

Beamforming Vector Selection (Encoding): For a given codebook $\mathcal{C}=\left\{\hat{v}_{1}, \ldots, \hat{v}_{N}\right\}$, the receiver encodes as follows ${ }^{2}$

$$
\hat{v}=\mathcal{Q}(v)=\arg \max _{\hat{v}_{i} \in \mathcal{C}}\left|\left\langle v, \hat{v}_{i}\right\rangle\right| .
$$

By the encoding scheme, the unit-norm hypersphere $\mathcal{S}_{t}=$ $\left\{u \in \mathbb{C}^{t}:\|u\|=1\right\}$, where random vector $v$ lies, is partitioned into $\left\{\overline{\mathcal{R}}_{i} ; i=1, \ldots, N\right\}$, where

$$
\overline{\mathcal{R}}_{i}=\left\{v \in \mathcal{S}_{t}:\left|\left\langle v, \hat{v}_{i}\right\rangle\right| \geq\left|\left\langle v, \hat{v}_{j}\right\rangle\right|, \forall j \neq i\right\} .
$$

The encoding scheme (11) can be restated simply as $\mathcal{Q}(v)=$ $\hat{v}_{i}$ if $v \in \overline{\mathcal{R}}_{i}$.

The MSwIP design method can be further simplified so that it does not depend on $P_{T}$ (for details, see [4]). The paper [4] also presents results for a low-complexity VQ method, more specifically tree-structured VQ.

\section{Capacity Loss with Quantized Feedback}

In this section, we will quantify the effect of quantization of beamforming vector with a finite number of bits on the channel capacity for the MISO channel with i.i.d. $\tilde{\mathcal{N}}(0,1)$ entries. With a given number of feedback (quantization) bits, we want to know how close we can approach to the performance of the complete feedback. The capacity loss $C_{L}=$ $E_{h}\left[I_{L}(h, \mathcal{Q}(v))\right]$ will be analyzed in terms of $t$ and $B$. Since $\mathcal{Q}(v)=\hat{v}_{i} \forall h \in \mathcal{R}_{i}$ from (11), the capacity loss can be expressed as

$$
C_{L}=\sum_{i=1}^{N} P\left(h \in \mathcal{R}_{i}\right) E_{h \in \mathcal{R}_{i}}\left[-\log \left(1-\tilde{\alpha}^{2}\left(1-\left|\left\langle v, \hat{v}_{i}\right\rangle\right|^{2}\right)\right)\right] .
$$

A. Approximate Density Function of $|\langle v, \mathcal{Q}(v)\rangle|^{2}$

In (13), note that $h \in \mathcal{R}_{i}$ implies $v \in \overline{\mathcal{R}}_{i}$. In order to calculate (13), we need to obtain the conditional density of the inner product $\gamma_{i}:=\left|\left\langle v, \hat{v}_{i}\right\rangle\right|^{2}$ given $v \in \overline{\mathcal{R}}_{i}$. For that purpose, we start with a simpler related random variable, which is described in the following Lemma. In the following, $\beta(a, b)$ represents the beta distribution with parameters $(a, b)$.

Lemma 1: For $v \sim \mathcal{U}\left(\mathcal{S}_{t}\right)$ and a fixed $v_{0} \in \mathcal{S}_{t}$, the random variable $\gamma_{0}=\left|\left\langle v, v_{0}\right\rangle\right|^{2}$ has a $\beta(1, t-1)$ distribution. That is, the density function for $\gamma_{0}$ is given by

$$
f_{\gamma_{0}}(x)=(t-1)(1-x)^{t-2}, 0<x<1 .
$$

Similarly, $\xi_{0}=1-\gamma_{0}$ has a $\beta(t-1,1)$ distribution.

$$
f_{\xi_{0}}(x)=(t-1) x^{t-2}, 0<x<1 .
$$

Proof: See [6].

Now let us look at the conditional density of $\gamma_{i}=\left|\left\langle v, \hat{v}_{i}\right\rangle\right|^{2}$ given $v \in \overline{\mathcal{R}}_{i}$. From the optimum encoding given in (11)

\footnotetext{
${ }^{2}$ Encoding process in beamforming vector selection is denoted by $\mathcal{Q}(v)$ since it only depends on $v$, while in designing a codebook in (7) the encoding is also dependent on $\alpha$, hence, it is denoted by $\mathcal{Q}(h)$.
} 
or (12), generally each quantization cell has a complicated shape with boundaries defined by neighboring code vectors, and the quantization cells could be all different shapes. This geometrical complexity in the quantization cells makes it difficult to obtain the exact conditional density for $\gamma_{i}$ with a closed-form expression. Using intuitive arguments, we now develop an approximation to this conditional density.

When $N$ is reasonably large, since $v$ is uniformly distributed over $\mathcal{S}_{t}, P\left(v \in \overline{\mathcal{R}}_{i}\right) \simeq 1 / N$ for all $i$, and furthermore the shapes of quantization cells will be approximately identical. For analytical tractability, in this paper we constantly consider the following approximation for the quantization cell.

$$
\overline{\mathcal{R}}_{i} \simeq \tilde{\mathcal{R}}_{i}=\left\{v \in \mathcal{S}_{t}: 1-\left|\left\langle v, \hat{v}_{i}\right\rangle\right|^{2} \leq \delta\right\}
$$

for all $i$ and some $\delta>0$, which will be determined as a function of $B$. Under the large $N$ assumption, since $v \sim$ $\mathcal{U}\left(\mathcal{S}_{t}\right)$, it is reasonable to assume that $P\left(v \in \overline{\mathcal{R}}_{i}\right)=1 / N$ for all $i$. We solve for $\delta$ to satisfy the condition

$$
P\left(v \in \overline{\mathcal{R}}_{i}\right)=P\left(\gamma_{i} \geq 1-\delta\right)=\int_{1-\delta}^{1} f_{\gamma_{i}}(x) d x=\frac{1}{2^{B}} .
$$

Here note that with the quantization cell approximation of (16), when $v \in \tilde{\mathcal{R}}_{i}, \gamma_{i}$ has same density as $\gamma_{0}$. That is, $f_{\gamma_{i}}(x)=f_{\gamma_{0}}(x)$ for $1-\delta<x<1$. Then, using the density for $\gamma_{0}$ which was derived in Lemma 1, it can be easily shown that

$$
\delta=2^{-\frac{B}{t-1}} .
$$

Although generally there are overlaps in the approximated quantization cells, the analytical results from the approximation turn out to be quite accurate even when $N$ is small.

With the quantization cell approximation, since the approximated cells $\tilde{\mathcal{R}}_{i}$ have the identical geometrical shape and the probabilities $P\left(v \in \tilde{\mathcal{R}}_{i}\right)$ are all equal to $1 / 2^{B}$, the random variables $\gamma_{i}$ given $v \in \mathcal{R}_{i}$ have the same density of

$$
\begin{aligned}
f_{\gamma_{i}}\left(x \mid v \in \tilde{\mathcal{R}}_{i}\right) & =\frac{f_{\gamma_{0}}(x) 1_{[1-\delta, 1)}(x)}{P\left(v \in \tilde{\mathcal{R}}_{i}\right)} \\
& =2^{B} f_{\gamma_{0}}(x) 1_{[1-\delta, 1)}(x), \text { for all } i
\end{aligned}
$$

where $1_{A}(x)$ is the indicator function having value 1 if $x \in A$ and 0 otherwise. Therefore, we can focus on a particular quantization cell. As a result, we arrive at the following approximation for the density function.

Approximate Density: With the quantization cell approximation described in (16) and (17), the density function for $\gamma=$ $|\langle v, \mathcal{Q}(v)\rangle|^{2}$ is approximated by a truncated beta distribution

$$
f_{\gamma}(x) \simeq \tilde{f}_{\gamma}(x)=2^{B} f_{\gamma_{0}}(x) 1_{[1-\delta, 1)}(x) .
$$

Similarly, $\xi=1-\gamma$ is approximated by

$$
f_{\xi}(x) \simeq \tilde{f}_{\xi}(x)=2^{B} f_{\xi_{0}}(x) 1_{[0, \delta)}(x) .
$$

Fig. 1 shows the approximate densities of $\gamma$ for $t=3$ and various $B$, together with the densities obtained from simulations using codebooks designed by the MSwIP method.

\section{B. Performance Bounds Using the Approximate Density}

Here we quantify the nature of approximation and show that the quantization cell approximation discussed in Section IV-A leads to a lower bound on the capacity loss. The following Lemma is important in proving the main result.

Lemma 2: For $1-\delta \leq x<1, f_{\gamma}(x) \leq \tilde{f}_{\gamma}(x)$.

Proof: See [6].

Theorem 1: The capacity loss, $\tilde{C}_{L}$, that is obtained with the approximate density $\tilde{f}_{\gamma}$ is a lower bound on the actual capacity loss $C_{L}$ associated with the true density $f_{\gamma}$. That is

Proof: See Appendix.

$$
\tilde{C}_{L} \leq C_{L} \text {. }
$$

Theorem 1 can be generalized to any monotonically decreasing function in $\gamma$. For example, the outage probability and the symbol error probability that are obtained using $\tilde{f}_{\gamma}$ are also lower bounds on the actual performances.

\section{Capacity Loss with Quantized Beamforming}

Now returning to the original problem of capacity loss which we recall can be written as

$$
C_{L}=E\left[-\log \left(1-\frac{\alpha^{2} P_{T}}{1+\alpha^{2} P_{T}} \xi\right)\right] .
$$

Using the Taylor series expansion $-\log (1-x)=\sum_{k=1}^{\infty} x^{k} / k$, (21) can be expanded as

$$
C_{L}=\sum_{k=1}^{\infty} \frac{1}{k} E\left[\left(\frac{P_{T} \alpha^{2}}{1+P_{T} \alpha^{2}}\right)^{k}\right] E\left[\xi^{k}\right]
$$

where the independence of $\alpha$ with $v$, hence with $\xi$, is used.

First, using (17) and the approximate density given in (20), we have

$$
E\left[\xi^{k}\right]=\int_{0}^{\delta} x^{k} 2^{B}(t-1) x^{t-2} d x=\frac{t-1}{k+t-1} 2^{-\frac{k B}{t-1}} .
$$

To calculate the first expectation in (22), the density function for $\alpha^{2}$ is necessary. It is well-known that $\alpha^{2}=\|h\|^{2}$ has a gamma distribution [7]

$$
f_{\alpha^{2}}(x)=\frac{x^{t-1} e^{-x}}{\Gamma(t)}, x>0 .
$$

Using this, we can show that (see [6] for details)

$$
E\left[\left(\frac{P_{T} \alpha^{2}}{1+P_{T} \alpha^{2}}\right)^{k}\right]=\frac{\Gamma(k+t)}{\Gamma(t)} P_{T}{ }_{2}{ }_{2} F_{0}\left(k+t, k ; ;-P_{T}\right)
$$

where ${ }_{2} F_{0}$ is the generalized hypergeometric function ${ }_{p} F_{q}$ with $p=2$ and $q=0$. Putting (23) and (25) into (22), finally we have

$$
\tilde{C}_{L}=\frac{t-1}{\Gamma(t)} \sum_{k=1}^{\infty} \frac{\Gamma(k+t)}{k+t-1} 2^{-\frac{k B}{t-1}} P_{T}{ }_{2}{ }_{2} F_{0}\left(k+t, k ; ;-P_{T}\right) .
$$

From the expression in (26), we note that $\tilde{C}_{L}$ goes to zero as $B$ increases to infinity.

Approximation to the Capacity Loss: Consider $I_{L}(h, \hat{v})$ given in (5). When $|\langle v, \hat{v}\rangle| \simeq 1$ and $P_{T} \gg 1$ (called 
high-resolution and high-SNR assumption), $I_{L}(h, \hat{v})$ can be approximated as $I_{L}(h, \hat{v}) \simeq 1-|\langle v, \hat{v}\rangle|^{2}$. For the approximated capacity loss, we can simply take the expectation of $\xi=$ $1-|\langle v, \hat{v}\rangle|^{2}$ using its approximate density in (20).

$$
\tilde{C}_{L} \simeq 2^{B} \int_{0}^{\delta} x f_{\xi_{0}}(x) d x=\left(\frac{t-1}{t}\right) 2^{-\frac{B}{t-1}} .
$$

\section{Numerical Results and Discussion}

Fig. 2 shows the capacity loss for MISO systems for $t=2,3,4$ when the beamforming vector is quantized with $B=1, \ldots, 8$. The figure plots the exact analytical result $\tilde{C}_{L}$ given in (26) together with the simulation results using the codebooks designed with the MSwIP method. We can see that more quantization bits are required to meet a certain value of the capacity loss, e.g., $C_{L}=10^{-1}$, as the dimension increases. Also the slope of the curve decreases as $t$ increases.

\section{Outage Probability with Quantized Feedback}

Another important performance measure for fading channels is the outage probability, especially when the ergodicity requirement $T \gg T_{\text {coh }}$ (meaning that the transmission time is much larger than the channel coherence time) cannot be satisfied. In this section, the outage probability studied in [2] is analyzed by directly applying the distributions derived in Section IV. The outage probability for a given transmit power $P_{T}$ and rate $R$ (bits per channel use) is defined as

$$
P_{\text {out }}\left(R, P_{T}\right)=P[I(h, \hat{v})<R] .
$$

It can be rewritten in terms of $\alpha^{2}$ and $\gamma$ as

$$
P_{\text {out }}\left(R, P_{T}\right)=P\left(\alpha^{2} \gamma<\beta_{0}\right)=E_{\alpha^{2}}\left[P\left(\gamma<\frac{\beta_{0}}{\alpha^{2}} \mid \alpha^{2}\right)\right]
$$

where $\beta_{0}=\left(2^{R}-1\right) / P_{T}$.

Using the approximate density $\tilde{f}_{\gamma}$ in (19), the conditional probability in (28) is given by

$$
P\left(\gamma<\frac{\beta_{0}}{\alpha^{2}} \mid \alpha^{2}\right) \simeq\left\{\begin{array}{l}
1, \alpha^{2} \leq \beta_{0} \\
2^{B}\left[\delta^{t-1}-\left(1-\frac{\beta_{0}}{\alpha^{2}}\right)^{t-1}\right], \beta_{0}<\alpha^{2}<\beta_{1} \\
0, \alpha^{2} \geq \beta_{1}
\end{array}\right.
$$

where $\beta_{1}=\beta_{0} /(1-\delta)$. Then, by averaging (29) with respect to $f_{\alpha^{2}}$ given in (24), we finally arrive at

$$
P_{\text {out }}\left(R, P_{T}\right) \geq 1-2^{B} e^{-\beta_{0}}+e^{-\beta_{1}} \sum_{k=0}^{t-1} \frac{2^{B}\left(\beta_{1}-\beta_{0}\right)^{k}-\beta_{1}^{k}}{k !} \text {. }
$$

Interestingly, this bound is exactly the same as the one derived in [2].

\section{Bit ERror Probability with Quantized FEEDBACK}

In this section, the effect of quantization of the beamforming vector on the bit error probability will be analyzed. Consider a uncoded MISO system with transmit beamforming along $\hat{v}$. The received signal is represented as

$$
y=\alpha|\langle v, \hat{v}\rangle| s+\eta
$$

where $\eta \sim \tilde{\mathcal{N}}\left(0, \sigma_{\eta}^{2}\right)$. In this section, we present results for the case of binary signalling $s= \pm \sqrt{P_{T}}$ and coherent detection at the receiver. However, we would like to note that the approach introduced in this section is general and not limited to the binary case.

The probability of bit error for a given channel $h$ is

$$
P(E \mid h)=Q\left(\sqrt{2 \alpha^{2} \gamma \Gamma}\right)
$$

where $E$ is the error event, $Q(x)$ is the Q-function defined as $Q(x)=\frac{1}{\sqrt{2 \pi}} \int_{x}^{\infty} e^{-t^{2} / 2} d t, \gamma=|\langle v, \hat{v}\rangle|^{2}$ as before, and $\Gamma=$ $P_{T} / \sigma_{\eta}^{2}$ (transmit SNR). Taking an expectation over channel realizations and noting that two random variables $\alpha^{2}$ and $\gamma$ are independent, the bit error probability is given by

$$
P(E)=E_{h}[P(E \mid h)]=E_{\gamma}\left[E_{\alpha^{2} \mid \gamma}\left[P\left(E \mid \alpha^{2}, \gamma\right)\right]\right] .
$$

First, we consider the conditional probability of error for a given $\gamma$ denoted by $P(E \mid \gamma):=E_{\alpha^{2} \mid \gamma}\left[P\left(E \mid \alpha^{2}, \gamma\right)\right]$. Since $\alpha^{2}$ has the gamma distribution given in (24),

$$
\begin{aligned}
& P(E \mid \gamma)=\int_{0}^{\infty} Q\left(\sqrt{2 \alpha^{2} \gamma \Gamma}\right) f_{\alpha^{2}}\left(\alpha^{2}\right) d \alpha^{2} \\
& =\left(\frac{1-\mu(\gamma \Gamma)}{2}\right)^{t} \sum_{k=0}^{t-1}\left(\begin{array}{c}
t-1+k \\
k
\end{array}\right)\left(\frac{1+\mu(\gamma \Gamma)}{2}\right)^{k}
\end{aligned}
$$

where $\mu(a)=\sqrt{\frac{a}{1+a}}$ and (33) is a well-known result in the maximal ratio combining (MRC) and the maximal ratio transmission (MRT) analysis [7]. By taking an average over $\gamma$ using the density $\tilde{f}_{\gamma}$ derived in (19), we obtain a lower bound on the bit error probability

$$
\begin{aligned}
& \tilde{P}(E)=\frac{2^{B}(t-1)}{2^{t}} \sum_{k=0}^{t-1}\left(\begin{array}{c}
t-1+k \\
k
\end{array}\right) \frac{1}{2^{k}} \\
& \cdot \int_{1-\delta}^{1}(1-\mu(\gamma \Gamma))^{t}(1+\mu(\gamma \Gamma))^{k}(1-\gamma)^{t-2} d \gamma
\end{aligned}
$$

Fig. 3 shows the numerical and the simulation results for the bit error probability with varying number of transmit antennas $(t=1,2,3,4)$, and quantization bits $(B=2,4,8)$. The results for the perfect feedback, i.e., $B=\infty$, are also plotted for comparison. The lower bound appears to be very tight based on the simulation results.

\section{CONCLUSION}

We have investigated the problem of quantization associated with beamforming in multiple-antenna communication systems with finite-rate feedback. To analyze the performance of MISO systems with quantized beamforming, the i.i.d. Rayleigh fading case was considered and the density function of the squared inner-product between the optimum and the 
quantized beamforming vector was derived by considering an approximation of the quantization cell. Then, the density function was used to analyze the capacity loss due to quantization. The power of the approach was further demonstrated by applying the derived density function to analyze the outage probability and the bit error probability for a finite-rate feedback MISO system.

\section{APPENDIX: PROOF OF THEOREM 1}

Proof: We can write the capacity loss as an expectation over $\gamma$ and $\alpha^{2}$. That is,

$$
\begin{aligned}
C_{L} & =E_{h}\left[I_{L}(h, \hat{v})\right]=E_{\gamma, \alpha^{2}}\left[I_{L}\left(\alpha^{2}, \gamma\right)\right] \\
& =E_{\gamma}\left\{E_{\alpha^{2} \mid \gamma}\left[I_{L}\left(\alpha^{2}, \gamma\right)\right]\right\}
\end{aligned}
$$

where $I_{L}\left(\alpha^{2}, \gamma\right)=-\log \left(1-\frac{\alpha^{2} P_{T}}{1+\alpha^{2} P_{T}}(1-\gamma)\right)$. Let us define the conditional expectation inside the braces in (35) by $I_{L}(\gamma)$. Note that $I_{L}(\gamma)$ is a monotonically decreasing function in $\gamma$. Then, the Theorem can be proved as follows

$$
\begin{aligned}
C_{L}= & \int_{0}^{1} f_{\gamma}(\gamma) I_{L}(\gamma) d \gamma \\
= & \int_{1-\delta}^{1} \tilde{f}_{\gamma} I_{L} d \gamma-\int_{1-\delta}^{1}\left[\tilde{f}_{\gamma}-f_{\gamma}\right] I_{L} d \gamma+\int_{0}^{1-\delta} f_{\gamma} I_{L} d \gamma \\
\geq & \int_{1-\delta}^{1} \tilde{f}_{\gamma} I_{L} d \gamma-I_{L}(1-\delta) \int_{1-\delta}^{1}\left[\tilde{f}_{\gamma}-f_{\gamma}\right] d \gamma \\
& +I_{L}(1-\delta) \int_{0}^{1-\delta} f_{\gamma} d \gamma \\
= & \int_{1-\delta}^{1} \tilde{f}_{\gamma} I_{L} d \gamma+I_{L}(1-\delta)\left[\int_{0}^{1} f_{\gamma} d \gamma-\int_{1-\delta}^{1} \tilde{f}_{\gamma} d \gamma\right] \\
= & \int_{1-\delta}^{1} \tilde{f}_{\gamma}(\gamma) I_{L}(\gamma) d \gamma=\tilde{C}_{L}
\end{aligned}
$$

where (36) is because $\tilde{f}_{\gamma}(\gamma)-f_{\gamma}(\gamma) \geq 0$ for $1-\delta \leq \gamma<$ 1 from Lemma 2 and $I_{L}(\gamma)$ is a monotonically decreasing function in $\gamma$.

\section{ACKNOWLEDGMENT}

The authors would like to thank Chandra Murthy for his help in deriving the outage probability lower bound (30).

\section{REFERENCES}

[1] A. Narula, M. J. Lopez, M. D. Trott, and G. W. Wornell, "Efficient use of side information in multiple-antenna data transmission over fading channels," IEEE J. Select. Areas Commun., vol. 16, pp. 1423-1436, Oct. 1998.

[2] K. K. Mukkavilli, A. Sabharwal, E. Erkip, and B. Aazhang, "On beamforming with finite rate feedback in multiple antenna systems," IEEE Trans. Inform. Theory, vol. 49, no. 10, pp. 2562-2579, Oct. 2003.

[3] J. C. Roh and B. D. Rao, "Channel feedback quantization methods for MISO and MIMO systems," in Proc. IEEE PIMRC 2004, Barcelona, Spain, Sept. 2004.

[4] - "Vector quantization techniques for multiple-antenna channel information feedback," in Proc. Int. Conf. on Signal Processing and Commun. (SPCOM 2004), Dec. 2004.

[5] A. Gersho and R. M. Gray, Vector Quantization and Signal Compression. Kluwer Academic, 1992.

[6] J. C. Roh and B. D. Rao, "Transmit beamforming in multiple antenna systems with finite rate feedback: A VQ-based approach," IEEE Trans. Inform. Theory, submitted for publication.

[7] J. G. Proakis, Digital Communications, 3rd ed. McGraw-Hill, 1995.

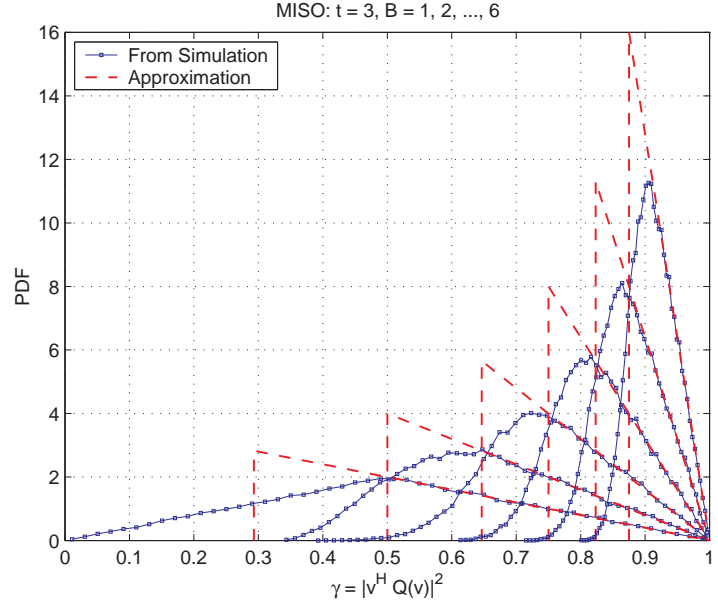

Fig. 1. Approximate density $\tilde{f}_{\gamma}(\gamma)(t=3$ and $B=1,2, \ldots, 6)$.

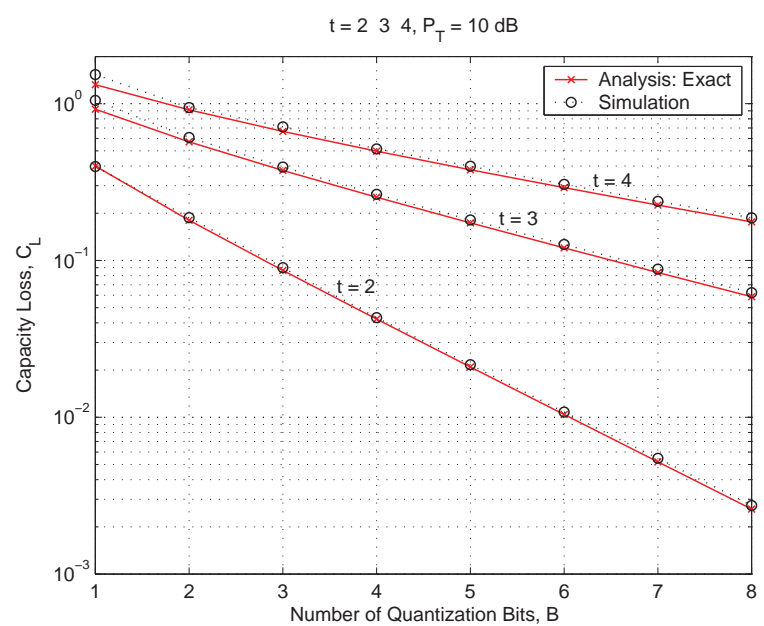

Fig. 2. Capacity loss due to quantization of beamforming vector $(t=2,3,4$; $\left.P_{T}=10 \mathrm{~dB} ; B=1,2, \ldots, 8\right)$. Here 'Exact' refers to $\tilde{C}_{L}$ given in (26).

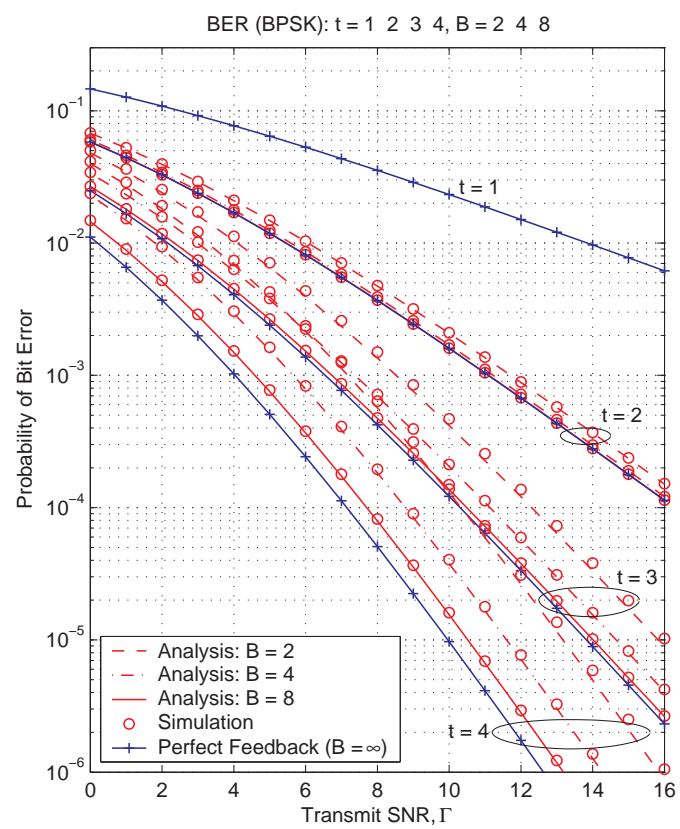

Fig. 3. Bit error probability of MISO systems with quantized beamforming $(t=1,2,3,4 ; B=2,4,8)$. 